\title{
Development of combined back propagation algorithm and radial basis function for diagnosing depression patients
}

\author{
R. Bhuvana ${ }^{1 *}$, S. Purushothaman ${ }^{2}$, R. Rajeswari ${ }^{3}$, R. G. Balaji ${ }^{4}$ \\ ${ }^{1}$ Bhuvana R., Assistant Professor, Department of Computer Science, A.M.Jain College, Chennai, India \\ ${ }^{2}$ Purushothaman S., Associate Professor, Institute of Technology, Haramaya University, Ethiopia \\ ${ }^{3}$ Rajeswari R., Lecturer, Institute of Technology, Haramaya University, Ethiopia \\ ${ }^{4}$ Balaji R.G., Lecturer, Institute of Technology, Haramaya University, Ethiopia \\ *Corresponding author E-mail: bhuvanavr1981@yahoo.co.in
}

Copyright $\odot 2015$ R.Bhuvana et al. This is an open access article distributed under the Creative Commons Attribution License, which permits unrestricted use, distribution, and reproduction in any medium, provided the original work is properly cited.

\begin{abstract}
Depression is a severe and well-known public health challenge. Depression is one of the most common psychological problems affecting nearly everyone either personally or through a family member. This paper proposes neural network algorithm for faster learning of depression data and classifying the depression. Implementation of neural networks methods for depression data mining using Back Propagation Algorithm (BPA) and Radial Basis Function (RBF) are presented. Experimental data were collected with 21 depression variables used as inputs for artificial neural network (ANN) and one desired category of depression as the output variable for training and testing proposed BPA/RBF algorithms. Using the data collected, the training patterns, and test patterns are obtained. The input patterns are preprocessed and presented to the input layer of BPA/RBF. The optimum number of nodes required in the hidden layer of $\mathrm{BPA} / \mathrm{RBF}$ is obtained, based on the change in the mean squared error dynamically, during the successive sets of iterations. The output of BPA is given as input to RBF. Through the combined topology, the work proves to be an efficient system for diagnosis of depression.
\end{abstract}

Keywords: Hamilton Rating Scale (HRS) Depression Data; BPA; RBF

\section{Introduction}

Depression can happen to everyone. It is a state of low mood and aversion to activity that can affect a person's thoughts, behavior, feelings and sense of well-being. Depressed people can feel sad, anxious, empty, hopeless, worried, helpless, worthless, guilty, alone, irritable, hurt, or restless. They may lose interest in activities that were once pleasurable, experience loss of appetite or overeating, have problems concentrating, remembering details or making decisions, and may contemplate, attempt or commit suicide. Insomnia, excessive sleeping, fatigue, aches, pains, digestive problems or reduced energy may also be present. Depressive episode involves symptoms such as depressed mood, loss of interest and enjoyment and increased fatigability. Depending upon the number of symptoms, depressive mood can be categorized as mild, moderate, or severe. Hybrid system (combination of different artificial neural networks, Fuzzy logic) have been used by Chattopadhyay [1-2], Subhrangsu [3].

Artificial neural networks (ANN) are powerful computational systems consisting of many simple processing elements connected together to perform tasks analogously to biological brains. They are making them efficient, robust, fault tolerant and noise independent. These can learn from training depression data and classify them into different categories. The decision-making process of ANN is based on the combination of entire input patterns, whereas the conventional mathematical system has to set about through the processing of individual data elements to arrive at a conclusion. The ANN can be organized into different topologies, such as feedforward and feedback networks. In this paper, we present two ANN algorithms: back propagation algorithm (BPA) and radial basis function (RBF) for categorizing and identifying the type of depression a patient has based 21 various inputs to the algorithm. 


\section{Literature survey}

Jason [4], presented three methods of performing pattern recognition on spatiotemporal plots produced by pharyngeal High-Resolution Manometry (HRM). The methods are classification models, including the Artificial Neural Networks (ANNs) Multi-Layer Perceptron (MLP) and Learning Vector Quantization (LVQ).

Antonio [5], presented a new approach to training Back-Propagation Artificial Neural Nets (BP-ANN) based on regularization and cross-validation and initialization by a Logistic Regression (LR) model. They have applied the approach to ten data sets of biomedical interest and systematically compared BP-ANN and LR.

Jyoti Joshi [6], mentioned that depression was a severe psychiatric disorder. Despite the high prevalence, current clinical practice depends almost exclusively on self-report and clinical opinion, risking a range of subjective biases. They focused on depression analysis based on visual cues from facial expressions and upper body movements.

Victor [7], implemented a neuro-fuzzy-Case Base Reasoning (CBR) driven decision support system that utilizes solutions to previous cases in assisting physicians in the diagnosis of depression disorder. The system represents depression disorder with 25 symptoms grouped into five categories. Fuzzy logic provided a means for handling imprecise symptoms. The five best-matched cases are subjected to the emotional filter of the system for diagnostic decision-making. This approach derived strengths from the hybridization since the tools are complementary to one another.

Subhagata [8], mentioned that depression was a common but worrying psychological disorder that adversely affects one's quality of life. It was more ominous to note that its incidence was increasing. In addition, grades of depression are often determined in continuous ranges, e.g., 'mild to moderate' and 'moderate to severe' instead of making them more discrete as 'mild', 'moderate', and 'severe'. They attempted to differentiate depression grades more accurately using two neural net learning approaches such as BPNN (Back Propagation Neural Network) and ANFISS (Adaptive Network-based Fuzzy Inference System).

Anish [9], implemented application of soft computing techniques to automate depression diagnosis. In order to achieve our goal, an intelligent Neuro-Fuzzy model has been developed. It has been trained with a sample of real-world depression data. Experiments with test data reveal that the Mean Squared Error in prediction was nominal for most of the cases. Such a system could assist the doctors to take decisions in much-needed situations.

Danuta [10], stated that family history of MDD increases individuals' vulnerability to depression and alters the way depression manifests itself. Emotion processing and attention shifting are functions altered by MDD and family history of the disease; therefore, it was important to recognize the neural correlates of these functions in association with both factors.

\section{Hamilton rating scale}

Hamilton depression rating scale, abbreviated HAM-D, is a multiple item questionnaires used to collect information from the affected patients. The scale provides an indication of depression, and as a guide, to evaluate recovery. Max Hamilton originally published the scale in 1960. Initially, the HAM scale was considered the "Gold Standard" for rating depression in clinical research.

Depression data from 1800 patients based on the Hamilton rating scale (HRS) using different questionnaire. The HRS has 21 categories of depression identified with each depression scaled in a range of 0 to 4 numerical values and a few categories are less than 4.The distribution of the collected data according is given in Table 1.

Table 1: Depression Data Grouped for Training and Testing BPA/RBF

\begin{tabular}{lllll}
\hline $\begin{array}{l}\text { Total Range Categorized in the } \\
\text { target values }\end{array}$ & $\begin{array}{l}\text { Target } \\
\text { Values }\end{array}$ & $\begin{array}{l}\text { No of Patterns in each } \\
\text { Category }\end{array}$ & $\begin{array}{l}\text { No of Patterns for } \\
\text { Training }\end{array}$ & $\begin{array}{l}\text { No of Patterns for } \\
\text { Testing }\end{array}$ \\
\hline$<15$ & 1 & 49 & 9 & 9 \\
$>=15$ and & 2 & 786 & 18 & 18 \\
$<30$ & & & & \\
$>=30$ and & 3 & 903 & 18 & 18 \\
$<45$ & 4 & 62 & 10 & 10 \\
$>45$ & & 1800 & 55 & 55 \\
Total & & & \\
\hline
\end{tabular}

\section{Back propagation algorithm (BPA) network}

This model is easy to understand, and can be implemented as a software simulation. BPA is a supervised method which maps inputs with target output. The BPA learns the statistical feature patterns. ANN using BPA has one input layer, one output layer. The number of nodes in the hidden layer of BPA is decided by pruning. 
Training BPA.

Forward propagation:

1) The weights of the ANN are initialized.

2) The inputs and outputs of training patterns are presented to the network. The output of each node in the successive layers is calculated.

3) The error $E(p)$ of a pattern is calculated as the difference between the actual output and the expected output.

The errors of all the training patterns are calculated as the Mean Squared Error (MSE), E (MSE) = E(p) . The activation function which is used to train the ANN is the sigmoid function and it is given by:

$$
f(x)=\frac{1}{1+\exp (-x)}
$$

Where:

$\mathrm{F}(\mathrm{x})$ is a nonlinear differentiable function.

Reverse propagation (Weight updation)

4) The error for the node in the output layer is calculated.

5) The weights between output layer and hidden layer are updated.

6) The errors for the nodes in the hidden layer is calculated

7) The weights between hidden layer and input layer are updated.

The above steps complete one weight updation. Second pattern is presented, and the above steps are followed for the second weight updating. When all the training patterns are presented, a cycle of iteration or epoch is completed.

Iterations versus MSE for BPA

The topology of the ANN is 21 nodes in the input layer, ten nodes in the hidden layer and one node in the output layer. When it reaches learning of all 55 training patterns by using randomly, then network going to stop and Mean Square Error can be found. Weight updation is done by presenting all the training patterns. The testing of the weight updation is done by presenting all the test patterns. The number of iterations, after which testing can be done, is fixed.

\section{Radial basis function (RBF)}

RBF Neural Network consists of an input layer and an output layer of nodes and a single hidden layer. A distance measure is used to associate the input with outputs. RBF is capable of performing approximations. The approximation is produced by passing an input pattern through a set of basic functions each of which contains one of the RBF centers. These are multiplied with the result of each function by a coefficient and then summing them linearly.

The activation function for RBF network is $\mathrm{f}(\mathrm{x})=\mathrm{e}^{-\mathrm{x}}$. The RBF learns sample 55 patterns out of 1800 patterns during training. It has an advantage that it does not involve in repeated training.

Training RBF

Step 1: Apply RBF.

$$
\begin{aligned}
& \text { No. of Input }=21 \\
& \text { No. of Patterns }=55 \\
& \text { No. of Centres }=10
\end{aligned}
$$

Step 2: Calculate $\mathrm{RBF}$ as $\mathrm{RBF}=\exp (-\mathrm{x})$

Step 3: Calculate Matrix as $\mathrm{G}=\mathrm{RBF} ; \quad \mathrm{A}=\mathrm{GT} * \mathrm{G}$

Step 4: Calculate $\quad B=A^{-1}$

Step 5: Calculate $\quad \mathrm{E}=\mathrm{B} * \mathrm{GT}$

Step 6: Calculate the final weight, $\quad \mathrm{F}=\mathrm{E} * \mathrm{D}$

Step 7: Store the final weights in a file.

Testing RBF

Step 1: Read the input

Step 2: Read the Final Weights

Step 3: Calculate Numerals $=\mathrm{F} * \mathrm{E}$

Step 4: Check the output with the templates for the type of depression. 


\section{Results and discussions}

Effect of centers in RBF performance

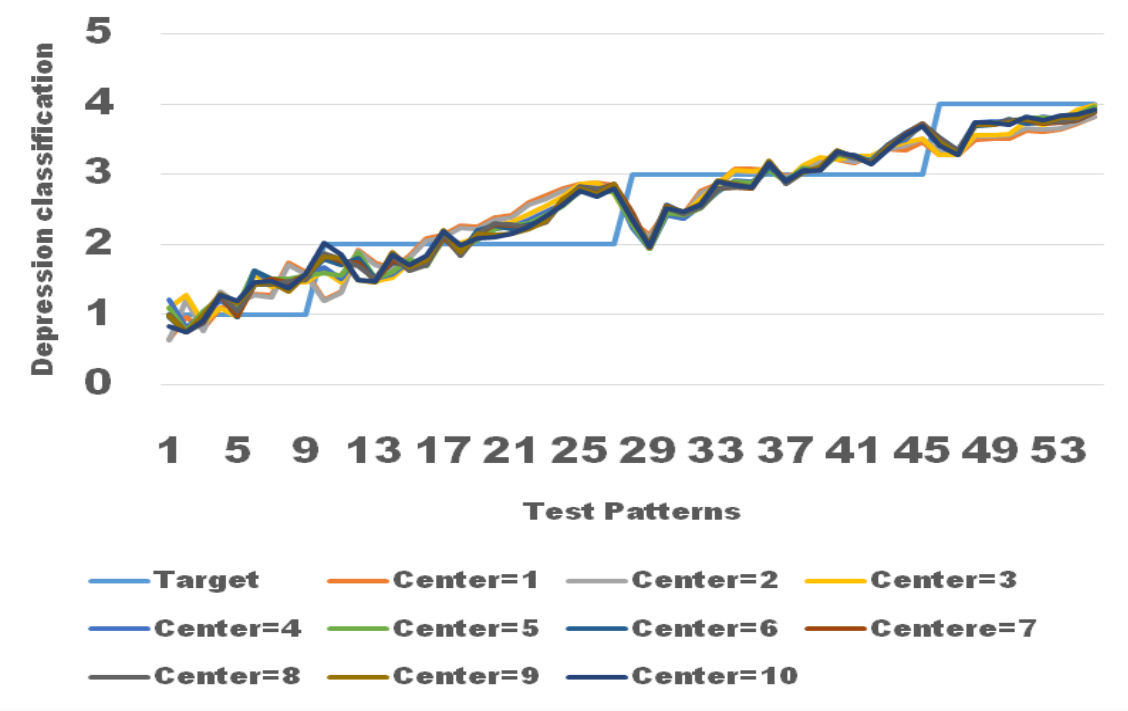

Fig. 1: Classification Output for Different RBF Centers

Figure 1 presents the outputs for 55 test patterns. The classification outputs of RBF with different number of centers are presented. The plot shows the performance of RBF when trained separately not combining with BPA. Estimation by RBF Network

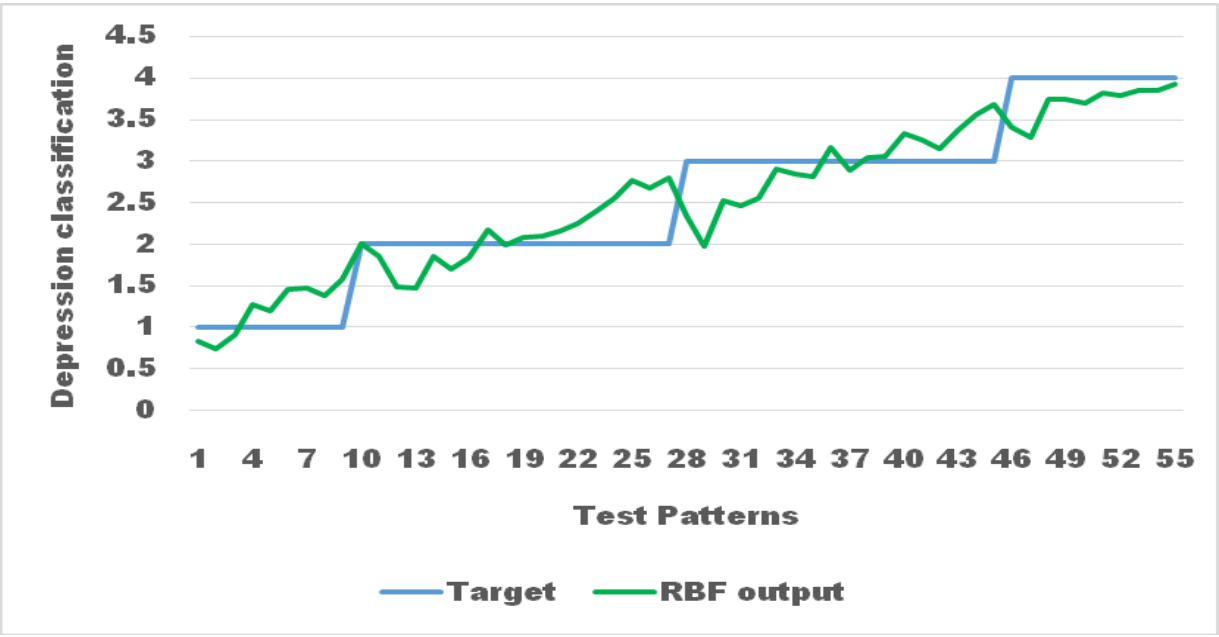

Fig. 2: RBF Classification Output

Figure 2 shows the output of RBF when trained separately not combining with BPA. Closer categorization by RBF is achieved when the number of centers is 10 in the hidden layer of RBF with 21 nodes in the input layer and one node in the output layer. The $\mathrm{x}$-axis shows the test pattern numbers and the $\mathrm{y}$-axis show the classification by RBF. The graph indicates the classified output is lying in the middle of the target output and hence the classification is acceptable.

\section{Depression detection and classification by BPA and RBF}

Combined approach of BPA \& RBF for depression detection (Figure 3)

Step 1: Depression features are presented in the input layer of BPA.

Step 2: Forward propagate the features through the hidden layer and to the output layer.

Step 3: The error is input to the input layer of the RBF.

Step 4: The depression category target is presented in the output layer of the RBF.

Step 5: The training process is continued till all the patterns are presented, and the summation of error in the output layer of the BPA is less than a specified value. 


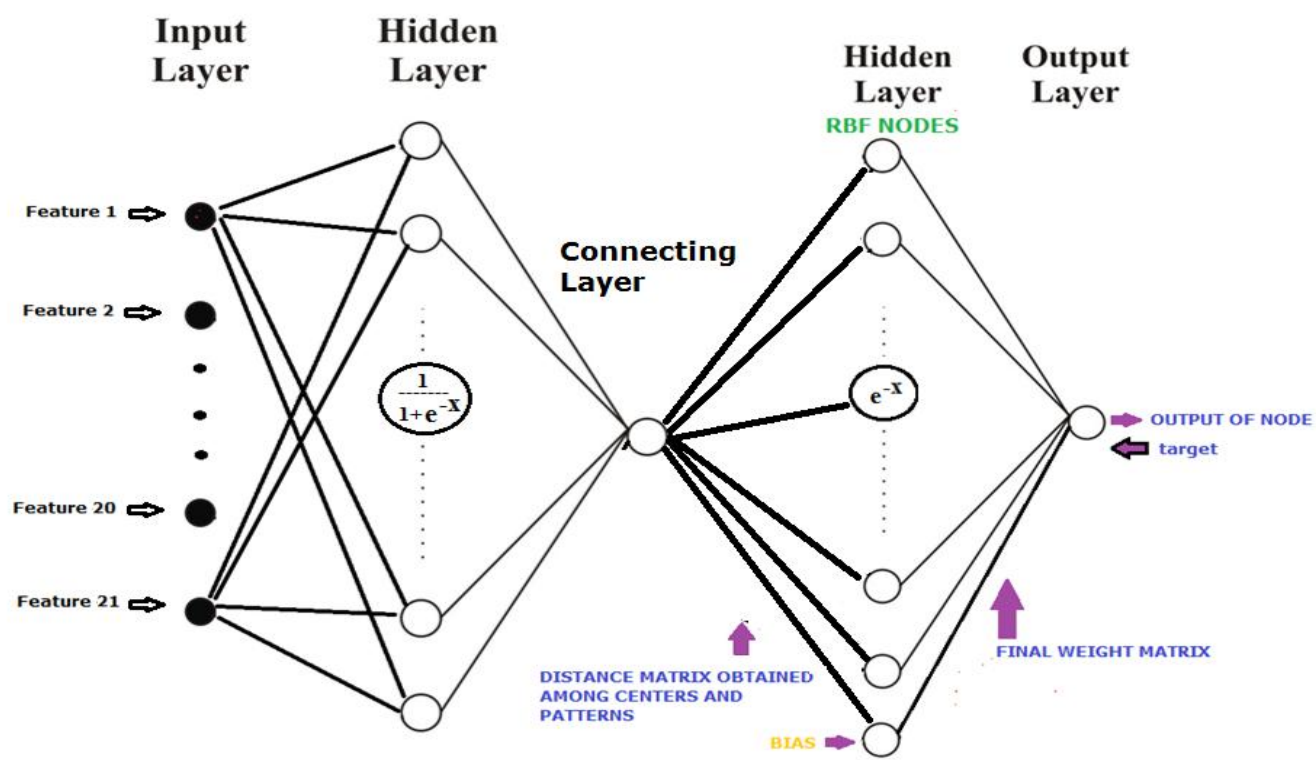

Fig. 3: Combined BPA and RBF Topology

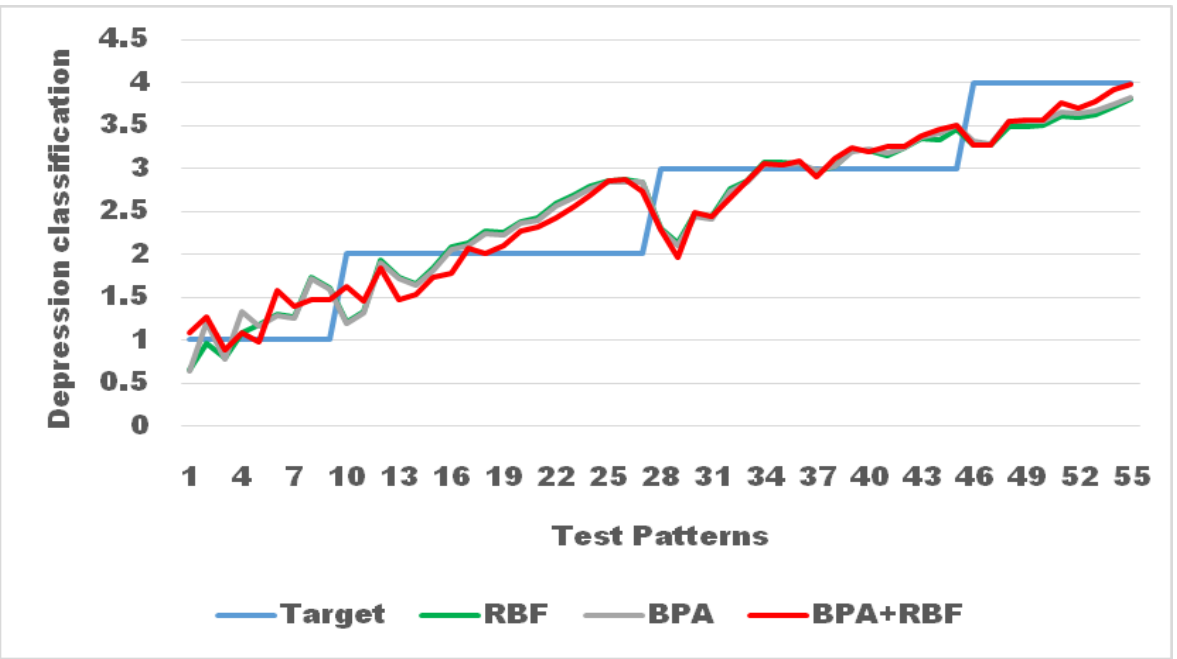

Fig. 4: Classification Performance of Combined Topology of BPA + RBF

By Figure 4, all 55 testing patterns are shown according to the category of depression.

\section{Conclusion}

Artificial neural network with supervised Back Propagation Algorithm (BPA) and Radial Basis Function (RBF) has been considered for depression classification. The performance of two algorithms is combined, and hence the error is minimized with more pattern classification correctly. The work proves to be an efficient system for diagnosis of depression. In the present work, data have been collected from a small group of depression affected patients. Different groups of patients have to be met. If there is a possibility, the collected data can be correlated with the commercial psychological database.

\section{References}

[1] S. Chattopadhyay, Neurofuzzy models to automate the grading of old-age depression, Expert Sys.: J. of Knowledge Engg., 31, 1, (2014), 4855 .

[2] S.Chattopadhyay, S.Banerjee, F.A.Rabhi, and R.U.Acharya, A Case-based Reasoning System for Complex Medical Diagnoses, Expert Systems: the Journal of Knowledge Engineering, 30, 1, (2013), 12-20.

[3] Subhrangsu Mukherjee, Kumar Ashish, Nirmal BaranHui, Subhagata Chattopadhyay, Modeling Depression Data: Feed Forward Neural Network vs. Radial Basis Function Neural Network, American Journal of Biomedical Sciences, American J. Biomed. Sci., 6, 3, (2014), 166174; doi: 10.5099/aj140300166. http://dx.doi.org/10.5099/aj140300166.

[4] Jason D. Mielens, R.Matthew, R.Hoffman Michelle, M.Ciucci Timothy, McCulloch, Jack J. Jiang, Application of Classification Models to Pharyngeal High-Resolution Manometry, Journal of Speech, Language, and Hearing Research, 55, (2012), 892-902. http://dx.doi.org/10.1044/1092-4388(2011/11-0088). 
[5] Antonio Ciampi, Fulin Zhang, A new approach to training back-propagation artificial neural networks: empirical evaluation on ten data sets from clinical studies, Statistics in Medicine, 21, 9, (2012), 1309-1330.

[6] Jyoti Joshi, AbhinavDhall, Roland Goecke., Michael Breakspear, and Gordon Parker, Neural-Net Classification For Spatio-Temporal Descriptor Based Depression Analysis, $21^{\text {st }}$ International Conference on Pattern Recognition, (2012), Tsukuba International Congress center, Japan.

[7] Victor E. Ekong, Udoinyang G Inyang, Emmanuel A. Onibere, Intelligent Decision Support System for Depression Diagnosis Based on Neuro-fuzzy-CBR Hybrid, Modern Applied Science, Vol.6, No.7. (2012), 79-88.

[8] SubhagataChattopadhyay, Preetisha Kaur, FethiRabhi, Rajendra Acharya U., Neural Network Approaches to Grade Adult Depression, Journal of Medical Systems, 36, 5, (2012), 2803-2815.

[9] Anish Dasari, NirmalBaranHui, Subhagata Chattopadhyay, A Neuro-Fuzzy System for Modeling the Depression Data, International Journal of Computer Applications, 54, 6, (2012), doi:10.5120/8567-2276. http://dx.doi.org/10.5120/8567-2276.

[10] Danuta M. Lisiecka, Angella Carballedo, Andrew J. Fagan, Yolande Ferguson, James Meaney, Thomas Frodl, Recruitment of the left hemispheric emotional attention neural network in risk for and protection from depression, Journal of Psychiatry Neuroscience, 38, 2, (2013), $117-28$. 